\title{
Analysis of Abstention in the Elections to the Catalan Parliament by Means of Decision Trees
}

\author{
Eva Armengol ${ }^{(凶)}$ and Zaida Vicente \\ Artificial Intelligence Research Institute (IIIA-CSIC), Campus UAB, \\ Camí de Can Planes, s/n, 08193 Barcelona, Bellaterra, Spain \\ eva@iiia.csic.es
}

\begin{abstract}
Democracies are based on political parties and election systems allowing voters to put the confidence in representers of these political parties to defend their interests. There are many studies analyzing the results of elections with the goal of (1) explaining the results, and (2) trying to predict what will happens in future elections. However no many attention has received the abstention, why voters do not use their right to elect representers? Commonly, abstention has not been too significant, however in last years it has been increased in many countries and it could be of great interest to analyze the causes. Studies about elections, both voting and abstention, are commonly based on statistical methods. The current paper is focused on analyzing the abstention based on symbolic learning methods (decision trees). Particularly, we are interested on identifying the groups of potential voters that have decided to abstain. We worked on data of the elections to Catalan Parliament held in 2015 .
\end{abstract}

Keywords: Electoral results $\cdot$ Ecological inference $\cdot$ Abstention $\cdot$ Explanations $\cdot$ Inductive learning methods $\cdot$ Decision trees

\section{Introduction}

One of the main rights in democracy is the free election of political representers. Representers belong to political parties that have a political program and voters choose the one that best fits their social, economic, and ethic values. Until recent times, political parties are aware to both predict the vote to their own party and, after the elections, explain why voters exhibited the resulting behaviour. Traditionally, predictions are made based on questionnaires before and during elections. In addition to directly ask for the vote, sometimes the voter is also questioned about aspects of his life. As McConway [13] explains, such questionnaires are difficult to elaborate and there is also a risk that the person asked do not answer what he actually believes (specially when asked directly for vote intention). 
However, in addition to the interest in determining the vote to each political party, another important fact arises: the abstention. Lastly, the percentage of voters that abstain in elections increases in all the countries. Ferreira et al. [4] analyzed the causes of this increment and mainly they associate it to political disaffection and no representativeness of political parties. In fact it is increasing the percentage of population that does not identify theirselves with a particular party [8]. There are several reasons for this lack of representativeness, for instance, lack of confidence in democracy in general, corruption, etc. [1,2,14].

Cazorla et al. [2] analyze the causes of abstention of Spanish voters in European elections held in 2014. In this analysis the authors conclude that in the particular case of Spain, there are two main reasons for the increment of abstention: (1) the fragmentation of the space of political parties mainly due to the growing of two new parties, and (2) the demobilisation and dissatisfaction of the voters, mainly due to economic crisis, corruption cases and political scepticism. This analysis has been made using the results of a post-electoral questionnaire designed to provide relevant information about both abstentionists and supporters of the different political parties. By grouping and recoding variables they used Structural Equation Modelling to obtain a set of variables useful to explain abstention.

Ecological inference [10] is the process to use aggregated data to infer relationships at individual level when there are not information about individuals. The ecological inference handles two observed variables $X_{i}$ and $Y_{i}$ and two unobserved variables $\alpha_{i}$ and $\beta_{i}$ for $1 \leq i \leq n$ being $n$ the number of observations (aggregated units). Until 1997 there were two main approaches for solving ecological inference problems: the followers of the method of bounds proposed by Duncan y Davis [3], and the followers of the Goodman's approach, based on regression [7]. In 1997, King [9] introduced a new paradigm based on both approaches consisting on, given a set of constraints (see [10]) the goal is to obtain the straight lines that he calls tomographies that represent the space of possible solutions. Such space represents an estimation of the parameters $\alpha_{i}$ and $\beta_{i}$ of the ecological inference.

Castela and Galindo-Villardón [1] use the ecological inference method proposed by King as a basis to use the HJ-Biplot method [19] to determine groups of population and their electoral behaviour from data of Portuguese elections held in 2002 and 2005. This work is interesting because it is possible to determine the evolution of votes. In our case we have only data belonging to one electoral date (the one of 2015 to Catalan Parliament).

A different approach is the one of Nwankwo et al. [14] that, from a questionnaire including socio-demographic questions, use Principal Component Analysis (PCA) to determine the main aspects that influence the abstention in South Eastern Nigeria. In particular, they obtained eight main components: socioeconomic status, lack of trust in the electoral process, social trust and unemployment, registration and demographic factor, corruption and inadequate security, deception and intimidation, indigene status and electoral manipulation and poverty. 
A method that has provided interesting results is the one proposed by Flaxman et al. [6]. In particular, it has been used to explain the results of the E.E.U.U. elections held in 2012 [6] and 2016 [5]. The Flaxman's method exploits the connexion between Gaussian process regression [17] and kernel ridge regression [18] to use Bayesian approximation for learning and to include prior information.

In this paper we introduce a different approach: the use of symbolic learning methods to analyze the results of elections. Statistical methods give us valuable information such as correlations between variables. However, correlations take globally all the data and in our opinion to provide a more detailed exam of the data it is necessary to fragment them in significant groups that follow a pattern. This kind of pattern is the one that can be obtained using decision trees $[15,16]$. The advantage of decision trees is that their results are easily understood by experts and can be seen as explanations or descriptions of classes of objects. Notice that, in fact, the goal of methods such as the ones of Flaxman, King, Duncan and Davis, and Goodman is to solve the problem on ecological inference, i.e., to obtain particular data from general data. Instead, our goal is almost the converse one, we want to obtain significant patterns (i.e., general enough) to describe population groups having a similar behaviour from the point of view of the abstention. However, we could obtain patterns satisfied by reduced groups of electoral sections allowing to solve the ecological inference problem. In other words, if we allow overfitting when growing the decision tree, we could obtain patterns satisfied by only a few of electoral sections that is the goal of ecological inference.

In Catalonia, there are four different kind of elections: local, Catalan Parliament, Spanish Parliament and European Parliament. If we analyze separately the results of each one of them, the patterns can be easily compared and it will be possible to show the differences or similarities of voters according each kind of election. We focus our analysis in the abstention. Thus, our goal is to characterize electoral sections according to their abstention level. In the future we plan to extend this analysis to other elections.

The paper is organized as follows. In Sect. 2 there is a brief explanation of decision trees. In Sect. 3 there is the description of the data base used in the experiments. Section 4 contains a description and a discussion of the experiments carried on. Finally, Sect. 5 is devoted to conclusions and future work.

\section{Decision Trees}

A Decision Tree (DT) is a directed acyclic graph in the form of a tree. The root of the tree has not incoming edges and the remaining ones have exactly one incoming edge. Nodes without outgoing edges are called leaf nodes and the others are internal nodes. A DT is a classifier expressed as a recursive partition of the set of known examples of a domain [12]. The goal is to create a domain model predictive enough to classify future unseen domain objects.

Each node of a tree has associated a set of examples that are those satisfying the path from the root to that node. The leaves determine a partition of the 
original set of examples, since each domain object only can be classified following one of the paths of the tree. The construction of a decision tree is performed by splitting the source set of examples into subsets based on an attribute-value test. This process is repeated on each derived subset in a recursive manner. Figure 1 shows the ID3 algorithm $[15,16]$ commonly used to grow decision trees. From a decision tree we can extract rules (i.e., patterns) giving descriptions of classes, since each path from the root to a leaf form a description of a class. When all the examples of a leaf belong to the same class such description is discriminant. Otherwise, the description is no discriminant.

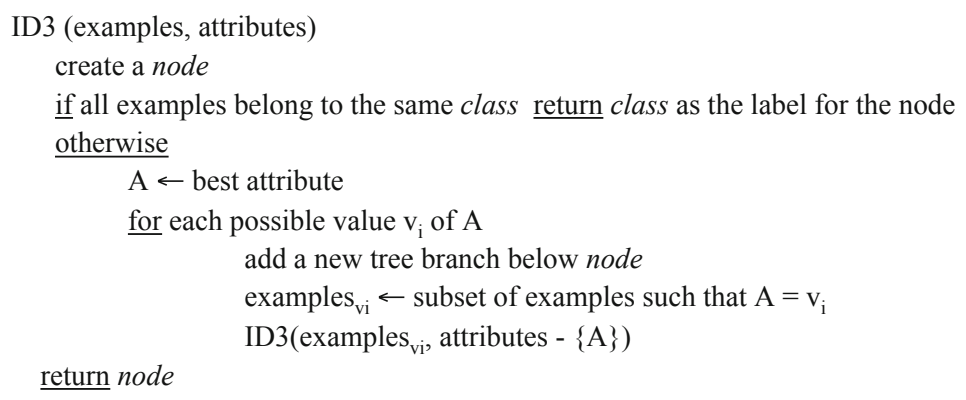

Fig. 1. ID3 algorithm for growing a decision tree.

A key issue of the construction of decision trees is the selection of the most relevant attribute to split a node. Each measure uses a different criteria, therefore the selected attribute could be different depending on it and thus the whole tree could also be different. In our experiments we used the López de Mántaras distance (LM) [11] that is an entropy-based normalized metric defined in the set of partitions of a finite set. It compares the partition induced by an attribute, say $a_{i}$, with the correct partition, i.e., the partition that classifies correctly all the known examples. The best attribute is the one inducing the partition which is closest to the correct partition. Given a finite set $X$ and a partition $\mathcal{P}=$ $\left\{P_{1}, \ldots, P_{n}\right\}$ of $X$ in $n$ sets, the entropy of $\mathcal{P}$ is defined as $(|\cdot|$ is the cardinality function):

$$
H(\mathcal{P})=-\sum_{i=1}^{n} p_{i} \cdot \log _{2} p_{i}, \text { where } p_{i}=\frac{\left|P_{i}\right|}{|X|}
$$

and where the function $x \cdot \log _{2} x$ is defined to be 0 when $x=0$. The López de Mántaras' distance between two partitions $\mathcal{P}=\left\{P_{1}, \ldots, P_{n}\right\}$ and $\mathcal{Q}=$ $\left\{Q_{1}, \ldots, Q_{m}\right\}$ is defined as:

$$
\operatorname{LM}(\mathcal{P}, \mathcal{Q})=\frac{H(\mathcal{P} \mid \mathcal{Q})+H(\mathcal{Q} \mid \mathcal{P})}{H(\mathcal{P} \cap \mathcal{Q})},
$$


where

$$
\begin{gathered}
H(\mathcal{P} \mid \mathcal{Q})=-\sum_{i=1}^{n} \sum_{j=1}^{m} r_{i j} \cdot \log _{2} \frac{r_{i j}}{q_{j}}, \quad H(\mathcal{Q} \mid \mathcal{P})=-\sum_{j=1}^{m} \sum_{i=1}^{n} r_{i j} \cdot \log _{2} \frac{r_{i j}}{p_{i}}, \\
H(\mathcal{P} \cap \mathcal{Q})=-\sum_{i=1}^{n} \sum_{j=1}^{m} r_{i j} \cdot \log _{2} r_{i j}, \\
\text { with } q_{j}=\frac{\left|Q_{j}\right|}{|X|}, \text { and } r_{i j}=\frac{\left|P_{i} \cap Q_{j}\right|}{|X|} .
\end{gathered}
$$

Decision trees can be useful for our purpose because their paths give us patterns describing classes of objects (electoral sections in our approach) in a user-friendly manner. One shortcoming of decision trees is overfitting, meaning that there are few objects in most of the leaves of the tree. In other words, paths are actually descriptions that poorly represent the domain. The main procedure to either avoid or reduce overfitting is by pruning the tree, i.e., under some conditions, a node is no longer expanded. However, this means that leaves can contain objects belonging to several classes and, therefore, paths do not represent discriminatory descriptions of classes, i.e., these descriptions are satisfied by objects of more than one class. In our approach, we managed overfitting by controlling the percentage of elements of each class. Let $S_{N}$ be the set of objects associated with an internal node $N$, the stopping condition in expanding $N$ (the if of the ID3 algorithm) holds when the percentage of objects in $S_{N}$ that belong to the majority class decreases in one of the children nodes. In such a situation, the node $N$ is considered as a leaf.

Notice, however, that allowing overfitting can produce results near to the goal of ecologic inference since the patterns obtained can be specific enough to almost identify particular objects.

\section{The Data Base}

Electoral landscape of Catalonia is formed by 5048 electoral sections each one of them composed of a minimum of 500 potential voters and a maximum of 2000 . Most of times, each electoral section corresponds to an electoral table although this can variate if the number of voters of a table is either too high or the population is scattered throughout the territory represented by the electoral section. In such cases, an electoral section is divided in several electoral tables with the constraint that no electoral table can have less than 200 voters.

The data base we have is composed of 5048 records, each one of them corresponds to an electoral section in Catalonia. Each record has two kinds of information: socio-demographic data and electoral data. Socio-demographic data has been formed from an aggregation of the data coming from several public data bases. The result of such aggregation is the typology of families seen in Table 1 and it is the one contained in the data base Habits ${ }^{\complement}$ from the AIS Group. The file containing the electoral results for all the Catalan electoral sections is public 
and contains (in absolute numbers and in percentage) the votes to each political party, the null votes, the blank votes, abstention and how many potential votes has an electoral section (electoral census). This file does not contain sociodemographic information associated to the electoral sections. Then, an ecological inference process has been carried out to assess how many families of each typology are in each electoral section. In https://www.ais-int.com/marketing-y-ventas/ geomarketing-habits-big-data/ there is a description of the method used to assess how many families of each typology are in each electoral section. The resulting file containing the socio-demographic information related to each electoral section is the one we used in our experiments.

Table 1. Families typology as obtained as it is explained in https://www.ais-int.com/ marketing-y-ventas/geomarketing-habits-big-data/

\begin{tabular}{l|l}
\hline Type & Description \\
\hline AF & Families with adolescents (until 18 years old) and children \\
\hline BG & Families with young sons (from 18 to 35 years old) \\
\hline CH & Families with children \\
\hline EI & Families where the main salary is a pension \\
\hline J & Families with two working persons and without children (DINK) \\
\hline LP & Families with one or two members, no children, only one salary \\
\hline $\mathrm{M}$ & Families with one or two members, no children, one or two pensions \\
\hline $\mathrm{N}$ & Singles older than 35 with a salary \\
\hline $\mathrm{O}$ & The main person is a student, a housework or a permanent disability \\
\hline $\mathrm{Q}$ & The main person of the family is unemployed \\
\hline $\mathrm{R}$ & Family formed by only one person receiving a pension \\
\hline Expenses & The main person of the family is an immigrant \\
\hline Income & Average of the income in a family \\
\hline
\end{tabular}

Table 2 shows the electoral attributes we used for each electoral section. Mainly there are percentages of both voters and votes to each political party, and we rejected all results corresponding to absolute numbers. We have take such decision in order to properly compare the results between political parties. The elections to the Catalan Parliament held in 2015 were somewhat special because of in addition to the traditional vote according right or left ideology, the independentist/no independentist aspect was very important. In fact, this aspect conducted to the creation of a new coalition and also was the focus of the electoral campaign. The main parties that concurred to the elections were the following:

- Cs (Ciudadanos). Party of right ideology and no independentist.

- JxSi (Junts pel Si). New independentist coalition formed by a right party and a left one.

- PSC (Socialist). Party of center-left ideology and no independentist. 
- CSQEP (Catalunya Si Que Es Pot). Coalition of left ideology and mainly no independentist although some members of it could be favorable to independence.

- PP (Partit Popular). Party of right ideology and no independentist.

- CUP (Candidatures d'Unitat Popular). Party of extreme left and anti-system ideology, and independentist.

All the attributes have numerical values and we have discretized them. Such discretization has been done by computing the quartiles of each one and assessing a label to each one of the resulting intervals. Table 2 shows the quartiles used to define the discretization intervals of the electoral attributes and the label we associated with them. The information about the families of each electoral section has been discretized in a similar way.

Summarizing, the data base used in the experiments is composed of the attributes shown in Tables 1 and 2 each one of them discretized according the corresponding quartiles.

Table 2. Discretization intervals for some the atributes corresponding to electoral results, i.e., the percentage of votes for null votes, blank votes, abstention, number of voters, and votes to each relevant political party.

\begin{tabular}{l|l|l|l|l}
\hline Attribute & Very low & Low & High & Very high \\
\hline Census & {$[25,796]$} & $(796,1040]$ & $(1040,1310]$ & $(1310,3670]$ \\
\hline Null & {$[0,0.15]$} & $(0.15,0.32]$ & $(0.32,0.54]$ & $(0.54,5.37]$ \\
\hline Blank & {$[0,0.29]$} & $(0.29,0.49]$ & $(0.49,0.72]$ & $(0.72,4.03]$ \\
\hline Abstention & {$[3.16,17.7]$} & $(17.7,21.6]$ & $(21.6,26.2]$ & $(26.2,73.3]$ \\
\hline Cs & {$[0,11]$} & $(11,17.3]$ & $(17.3,23.6]$ & $(23.6,50]$ \\
\hline JxSi & {$[2.45,24]$} & $(24,40.6]$ & $(40.6,54.2]$ & $(54.2,91.3]$ \\
\hline PSC & {$[0,7.19]$} & $(7.19,11.2]$ & $(11.2,17.5]$ & $(17.5,58.3]$ \\
\hline CSQEP & {$[0,4.67]$} & $(4.67,8.37]$ & $(8.37,12.4]$ & $(12.4,27]$ \\
\hline PP & {$[0,5.56]$} & $(5.56,7.87]$ & $(7.87,10.6]$ & $(10.6,42.1]$ \\
\hline CUP & {$[0,5.82]$} & $(5.82,7.87]$ & $(7.87,10.3]$ & $(10.3,47.7]$ \\
\hline
\end{tabular}

\section{Experiments}

Because our focus is the analysis of the abstention in the elections to Catalan Parliament, we have growth a decision tree taking as class label the percentage of abstention of the electoral sections. In order to avoid overfitting we cut the node expansion when the percentage of the majority class decreases if a node was expanded (see Sect. 2). We only have manually analyzed the paths (patterns) corresponding to leaves that contain around the $25 \%$ of the population in the root of the tree. Most of significant patterns are not discriminant, i.e., they are satisfied by electoral sections having different abstention levels.

After growing a decision tree, two patterns involving around the $25 \%$ of the electoral sections have been found: 
- $P 1:[[\mathrm{PSC}=$ very high $]]$

- $P 2$ : [[PSC = very low $]]$

We expected that the abstention was related with socio-demographic attributes as it has been found for instance in $[2,4]$. However, our experiments show that in the particular case of the Catalan elections held in 2015 the abstention level is directly related to the percentage of votes to the PSC party. In fact, this is not a contradictory result with the ones in $[2,4]$ since they only take into account the sociologic data coming from a questionnaire and no electoral data are included in their analysis. In our experiments, we included both socio-demographic data and electoral data. Moreover, our socio-demographic data come from real population data (census, register, and so on) instead of with a questionnaire.

Table 3. Level of abstention satisfied by the patterns $P 1, P 2$ and $P 3$ and both number (\#ES) and percentage (\%ES) of electoral sections satisfying the patterns.

\begin{tabular}{l|r|l|l|l|l|l}
\hline Pattern & \#ES & \%ES & Very low & Low & High & Very high \\
\hline$P 1$ & 1262 & 25.00 & 3.49 & 13.31 & 29.79 & 53.41 \\
\hline$P 2$ & 1263 & 25.02 & 60.25 & 25.18 & 10.85 & 3.72 \\
\hline$P 3$ & 976 & 19.33 & 15.06 & 32.58 & 31.97 & 20.39 \\
\hline
\end{tabular}

Table 3 shows the support of each one of the patterns $P 1$ and $P 2$ to each abstention level. Notice that around the $83 \%$ of electoral sections that have had many votes for PSC have been high or very high abstention levels (pattern $P 1$ ), the dual pattern (pattern P2) is also true: around the $85 \%$ of electoral sections that have had very few votes for PSC have been low or very low abstention levels. In other words, it seems that the level of abstention is directly correlated with the percentage of votes for PSC. In fact, this correlation has been proved statistically to be 0.580 . Also we have seen that the percentage of abstention has an inverse correlation of -0.546 with the percentage of votes for $\mathrm{JxSi}$. In our approach only the direct relations are explicit in the patterns, however a manual study of the patterns has show that the percentage of votes for PSC is inverse to the percentage of votes for $\mathrm{J} \times \mathrm{Si}$. That is to say, electoral sections with low percentage of votes for PSC have high percentage of votes to JxSi (and low percentage of abstention). In our opinion the relatively low correlation between PSC and abstention is due to the fact that the percentage of votes for PSC explains well the abstention for the extreme values (very low and very high), whereas in the intermediate part we cannot found any relevant pattern because there is not a significant trend and is this part the one responsible of the relatively low correlation. With our approach we are capable to take several subsets with different behaviour.

Patterns including intermediate levels of votes (high or low) to PSC are not significant enough to represent a clear abstention level. For instance the pattern $P 3=[$ PSC $=$ high] satisfied by around the $19 \%$ of electoral sections has not a clear support to any of abstention levels (see Table 3 ). Notice that around the $52 \%$ of electoral tables satisfying $P 3$ have high or very high abstention levels whereas the remaining $47.64 \%$ of electoral sections have low or very low abstention levels.

It has been surprising that information of families seems not to be relevant to explain the abstention level. In fact, we obtained some patterns relating the abstention with some family typology, for instance the following ones: 
- P4: [[PSC $=$ low $],[\mathrm{R}=$ high $]]$

- P5: [[PSC =low], [R =low]]

Notice that the patterns above relate low percentage of votes for PSC and the typology R (immigration) with a low level of abstention (around $65 \%$ and $77 \%$ respectively). Table 4 shows the support of the patterns $P 4$ and $P 5$ to each abstention level. These patterns are not significant enough due to low number of electoral sections that satisfy it, but we think that could be interesting as a basis to perform ecological inference. In the regression model also happens that the familiar information is not relevant to explain the abstention and we also found that families type $\mathrm{R}$ are the most correlated with the abstention. As future work, we have to check if this irrelevance of the family status is due to the way in which the family typologies has been obtained or conversely there is an actual independence between abstention and family status.

Table 4. Level of abstention satisfied by the patterns $P 4$ and $P 5$ and both number (\#ES) and percentage (\%ES) of electoral sections satisfying the patterns.

\begin{tabular}{l|l|l|l|l|l|l}
\hline Pattern & \#ES & \%ES & Very low & Low & High & Very high \\
\hline$P 4$ & 237 & 4.70 & 20.68 & 44.30 & 26.58 & 8.44 \\
\hline$P 5$ & 201 & 3.98 & 44.78 & 32.34 & 18.41 & 4.48 \\
\hline
\end{tabular}

With the goal of trying to relate abstention to family typologies, we have growth a decision tree taking into account only the attributes describing family typologies (those in Table 1). Most of the patterns are not significant in the sense that they are satisfied by only a few electoral sections. However, there are two patterns satisfied by a significant number of electoral sections (more than $25 \%$ ):

- $[[\mathrm{R}=$ very low]]: satisfied by 1262 electoral sections (25\%) where the $44.14 \%$ of them have very low abstention, the $25.59 \%$ low, the $18.86 \%$ high, and the $11.41 \%$ very high.

- $[[\mathrm{R}=$ high $]]$ : satisfied by 1240 electoral sections $(24.56 \%)$ where the $8.06 \%$ of them have very low abstention, the $17.50 \%$ low, the $27.82 \%$ high, and the $46.61 \%$ very high.

Thus, patterns above shows some trend to relate electoral sections with very low or low percentage of families type $\mathrm{R}$ with low or very low abstention (69.73\%) and electoral sections with high or very high percentage of families type $\mathrm{R}$ with high or very high abstention (74.43\%). Both patterns seems to support the idea that immigrant collective tends to abstain.

Summarizing, the results provided by decision trees are consistent with those obtained by statistical models. However, our approach is more informative since in addition to explicit the correlation between variables also explain the intervals where such correlation is higher. Thus, from the patterns we have seen that the percentage of votes to PSC is directly correlated with the percentage of abstention, but only when the percentage of votes is in one of the extremes, i.e., it is very low or very high, whereas in the intermediate values such correlation is not so clear. The statistical model gives a global perspective of the correlation between variables but it cannot focus on intervals as the patterns can do. 


\section{Conclusions and Future Work}

In this paper we introduced a new approach to analyze electoral data: the decision trees. This kind of methods are commonly used to construct domain models useful for prediction. In the current application, the branches of trees can be interpreted as patterns, i.e., similarities between the electoral sections that have a particular behaviour. Our focus has been the abstention and the goal was to show if electoral sections with similar abstention levels have also some common pattern. Experimental results have shown that patterns allow to separate subgroups of electoral sections (differently than statistical methods that have a global vision of them) and support the experts in focusing on a particular group and investigate in depth the characteristics of it.

Our main conclusions are that abstention level in Catalan elections held in 2015 was directly related with the percentage of votes to the Catalan Socialist Party (PSC). When we focus on sociologic features, we found that the percentage of families belonging to the typology R (immigration) is directly correlated with abstention.

In the future we plan to use the same methods on data coming from different Catalan Parliament elections. The patterns of both elections will be easy to compare and the experts could determine similarities and differences of behaviour of the same subgroup of electoral sections in different elections. A different research line could be to use the patterns in combination with some ecological inference method, such as the one of G. King.

Acknowledgments. The authors acknowledge the AIS Group enterprise (https:// www.ais-int.com/marketing-y-ventas/geomarketing-habits-big-data/) for having given us the Habits ${ }^{\circledR}$ Data Base in a selfless way. This research is funded by the project RPREF (CSIC Intramural 201650E044); and the grant 2014-SGR-118 from the Generalitat de Catalunya. Authors also thank to Àngel García-Cerdaña his helpful comments.

\section{References}

1. Castela, E., Galindo Villardón, M.P.: Inferencia ecológica para la caracterización de abstencionistas: El caso de portugal (in Spanish). Spat. Organ. Dyn. 3, 6-25 (2011)

2. Cazorla-Martín, A., Rivera-Otero, J.M., Jaráiz-Gulías, E.: Structural analysis of electoral abstention in the 2014 european parliamentary elections. Revista Española de Investigaciones Sociológicas, pp. 31-50 (2017)

3. Duncan, O.D., Davis, B.: An alternative to ecological correlation. Am. Sociol. Rev. 18, 665-66 (1953)

4. Ferreira, P., Dionisio, A.: Voters' dissatisfaction, abstention and entropy: analysis in european countries. https://www.researchgate.net/publication/23524254_Voters \%27_dissatisfaction_abstention_and_entropy_analysis_in_European_countries, 022008

5. Flaxman, S., Sutherland, D., Wang, Y.X., Whye Teh, Y.: Understanding the 2016 US Presidential Election using ecological inference and distribution regression with census microdata. arXiv e-prints, page arXiv:1611.03787 (2016)

6. Flaxman, S., Wang, Y.X., Smola, A.J.: Who supported obama in 2012? ecological inference through distribution regression. In: Proceedings of the 21th ACM SIGKDD International Conference on Knowledge Discovery and Data Mining, KDD 2015, pp. 289-298. New York, ACM (2015) 
7. Goodman, L.: Some alternatives to ecological correlation. Am. J. Sociol. - 64, 05 (1959)

8. Holland, I., Miskin, S.: Interpreting election results in western democracies. Current Issues Brief no.2 2002-03. Politics and Public Administration Group. Parliament of Australia. https://www.aph.gov.au/About_Parliament/ Parliamentary_Departments/Parliamentary_Library/Publications_Archive/CIB/ cib0203/03CIB02,2002

9. King, G.: A Solution to the Ecological Inference Problem. Princeton University Press, New Jersey (1997)

10. King, G., Rosen, O., Tanner, M.A. (eds.): Ecological Inference: New Methodological Strategies. Cambridge University Press, New York (2004). http://gking. harvard.edu/files/abs/ecinf04-abs.shtml

11. López de Mántaras, R.: A distance-based attribute selection measure for decision tree induction. Mach. Learn. 6, 81-92 (1991)

12. Maimon, O., Rokach, L. (eds.): Data Mining and Knowledge Discovery Handbook, 2nd edn. Springer, Berlin (2010). https://doi.org/10.1007/978-0-387-09823-4

13. McConway, K.: Explainer: how do you read an election poll? The Conversation. https://theconversation.com/explainer-how-do-you-read-an-election-poll$41204(2015)$

14. Nwankwo, C., Okafor, U., Asuoha, G.: Principal component analysis of factors determining voter abstention in south eastern nigeria. J. Pan Afr. Stud. 10, 249-273 (2017)

15. Quinlan, J.R.: Discovering rules by induction from large collection of examples. In: Michie, D. (ed.) Expert Systems in the Microelectronic Age, pp. 168-201. Edimburg Eniversity Press, Edinburgh (1979)

16. Quinlan, J.R.: Induction of decision trees. Mach. Learn. 1(1), 81-106 (1986)

17. Rasmussen, C.E., Williams, C.K.I.: Gaussian Processes for Machine Learning. The MIT Press, Cambridge (2006)

18. Saunders, C., Gammerman, A., Vovk, V.: Ridge regression learning algorithm in dual variables. In: Proceedings ot the 15th International Conference on Machine Learning, ICML (1998)

19. Galindo Villardón, M.P.: Una alternativa de representación simultánea: Hj-biplot (in Spanish). Questiio 10, 13-23 (1986) 